Revue internationale P.M.E.

Économie et gestion de la petite et moyenne entreprise

\title{
La contribution de la théorie des réseaux sociaux à la reconnaissance des opportunités de marché
}

\section{Didier Chabaud et Joseph Ngijol}

Volume 18, numéro 1, 2005

URI : https://id.erudit.org/iderudit/1008469ar

DOI : https://doi.org/10.7202/1008469ar

Aller au sommaire du numéro

Éditeur(s)

Presses de l’Université du Québec

ISSN

0776-5436 (imprimé)

1918-9699 (numérique)

Découvrir la revue

Citer cet article

Chabaud, D. \& Ngijol, J. (2005). La contribution de la théorie des réseaux sociaux à la reconnaissance des opportunités de marché. Revue internationale P.M.E., 18(1), 29-46. https://doi.org/10.7202/1008469ar

\section{Résumé de l'article}

Les travaux de recherche sur l'entrepreneuriat se sont multipliés ces dernières années. Néanmoins, l'analyse de la genèse du projet de l'entrepreneur est relativement peu développée. Cette étude vise à tracer les lignes d'un programme de recherche focalisé sur les stades d'avant-projet et de démarrage de l'activité de l'entreprise. Après une première partie faisant le point sur la littérature théorique et empirique qui existe sur la phase de reconnaissance d'opportunité par l'entrepreneur, nous procédons à une mise en perspective à l'aide de la théorie des réseaux sociaux. Cette théorie, issue notamment des travaux de Burt et de Granovetter, permet de souligner le rôle des réseaux sociaux dans la détection et dans l'évaluation des opportunités entrepreneuriales et ouvre la voie à des études empiriques approfondies sur le comportement de l'entrepreneur. 


\title{
La contribution de la théorie des réseaux sociaux à la reconnaissance des opportunités de marché
}

Didier CHABAUD

Joseph NGIJOL

Université de Cergy-Pontoise

\section{MOTS CLÉS}

Reconnaissance d'opportunité - Théorie des réseaux sociaux École autrichienne - Opportunité entrepreneuriale - Liens faibles

Trous structuraux

\begin{abstract}
RÉSUMÉ
Les travaux de recherche sur l'entrepreneuriat se sont multipliés ces dernières années. Néanmoins, l'analyse de la genèse du projet de l'entrepreneur est relativement peu développée. Cette étude vise à tracer les lignes d'un programme de recherche focalisé sur les stades d'avant-projet et de démarrage de l'activité de l'entreprise. Après une première partie faisant le point sur la littérature théorique et
\end{abstract}

\section{LES AUTEURS}

Didier Chabaud est maître de conférences en sciences de gestion à l'Université de CergyPontoise et professeur affilié à l'École de management de Normandie. Ses recherches appliquent la théorie des organisations (notamment, la théorie des réseaux sociaux et la théorie des coûts de transaction) à l'étude de la dynamique entrepreneuriale et de l'organisation du travail. Adresse: Université de Cergy-Pontoise, UFR d'économie et de gestion, 33, bd du Port, 95011 Cergy-Pontoise Cédex, France. Courriel: <Didier.Chabaud@eco.u-cergy.fr>.

JOSEPH NGIJOL est enseignant titulaire en stratégie à l'Université de Cergy-Pontoise. Ses recherches portent sur l'entrepreneuriat et en particulier sur les stratégies et comportements d'innovation des créateurs d'entreprise. Cet article est issu de son travail doctoral réalisé dans le cadre du programme doctoral ESSEC (École supérieure des sciences économiques et commerciales) de l'Université Paris-Dauphine. Adresse: ESSEC Ventures, avenue BernardHirsch, B.P. 50105 Cergy, 95021 Cergy-Pontoise Cédex, France. Courriel: <ngijol@essec.fr>.

1. Nous remercions Pierre-André Julien ainsi que les rapporteurs anonymes pour leurs commentaires et suggestions, mais demeurons - bien évidemment - responsables des erreurs et omissions. Les auteurs ont contribué de façon égale et sont cités par ordre alphabétique.

(C) 2005 - Presses de l'Université du Québec

Édifice Le Delta I, 2875, boul. Laurier, bureau 450, Sainte-Foy, Québec G1V 2M2 • Tél.: (418) 657-4399 - www.puq.ca

Tiré de: Revue internationale P.M.E., vol. 18, $\mathrm{n}^{\circ} 1$, sous la direction de Louis Raymond PME1801N

Tous droits de reproduction, de traduction et d'adaptation réservés 
empirique qui existe sur la phase de reconnaissance d'opportunité par l'entrepreneur, nous procédons à une mise en perspective à l'aide de la théorie des réseaux sociaux. Cette théorie, issue notamment des travaux de Burt et de Granovetter, permet de souligner le rôle des réseaux sociaux dans la détection et dans l'évaluation des opportunités entrepreneuriales et ouvre la voie à des études empiriques approfondies sur le comportement de l'entrepreneur.

\begin{abstract}
The amount of research on enterpreneurship has increased heavily over the last few years. Nevertheless, hardly any of it has focused on an analysis of the genesis of the entrepreneur's project. This paper aims to establish the framework of a research program focused on the first steps of the entrepreneurial project, i.e. the pilot study and the launching of the firm. After dealing in our first part with the existing theoretical and empirical literature on the theme of opportunity recognition by the entrepreneur, we will use the social network theory in our second part to put things in perspective. This theory, initiated notably by Burt and Granovetter, will enable us to underline the role of social networks in the detection and assessment of entrepreneurial opportunities, paving the way for detailed empirical studies on the entrepreneur's behaviour.
\end{abstract}

\title{
RESUMEN
}

Los trabajos de investigación sobre el empresariado se han multiplicado estos ultimos años. Sin embargo el anàlisis de la génesis del proyecto del empresario esta relativamente poco desarrollada. Este estudio tiende a trazar las lineas de un programa de investigación focalizado sobre los estadios de pre-proyecto y de la puesta en marcha de la actividad de la empresa. Despues de haber, en primer lugar, analizado la literatura teorica y empirica que existe sobre la fase de reconocimiento de oportunidad por el empresario, procedemos a una puesta en perspectiva con la ayuda de la teoria de las redes sociales, esta teoria, iniciada particularmente por los trabajos de Burt y Granovetter, permite resaltar el rol de las redes sociales en la detección y en la evaluacion de las oportunidades empresariales y abre la via a los estudios empiricos intensificados sobre el comportamiento del empresario.

\section{ZUSAMMENFASSUNG}

Die Forschung zum Thema Unternehmertum hat in den letzten Jahren erheblich zugenommen. Trotzdem sind die Analysen rund um die Entstehung von unternehmerischen Aktivitäten noch wenig entwickelt. Diese Studie zielt darauf ab, die Vorstufen der Unternehmenstätigkeit zu analysieren. Ein erster Teil thematisiert den aktuellen Stand der theoretischen und empirischen Literatur über die Phase der Erkennung einer Geschäftsgelegenheit durch den Unternehmer. Nachher fokussieren wir uns auf die Rolle der sozialen Netze in diesem Zusammenhang. Die Theorie, erarbeitet durch Burt und Granovetter, erlaubt die Untersuchung der Rolle der sozialen Netze in der Erkennung und Bewertung von Unternehmergelegenheiten. Sie ebnet dadurch den Weg für ausführliche empirische Studien über das Verhalten des Unternehmers.

Revue internationale P.M.E., vol. 18, $\mathrm{n}^{\circ} 1,2005$

(C) 2005 - Presses de l'Université du Québec

Édifice Le Delta I, 2875, boul. Laurier, bureau 450, Sainte-Foy, Québec G1V 2M2 • Tél.: (418) 657-4399 - www.puq.ca

Tiré de: Revue internationale P.M.E., vol. 18, $\mathrm{n}^{\circ} 1$, sous la direction de Louis Raymond • PME1801N

Tous droits de reproduction, de traduction et d'adaptation réservés 


\section{Introduction}

Comment l'entrepreneur fait-il pour reconnaître une opportunité de marché lui paraissant attractive au point de l'inciter à créer une entreprise? Cette question, qui apparaît comme première pour saisir la dynamique du processus entrepreneurial, a paradoxalement été peu étudiée par les économistes et les chercheurs en sciences de gestion. Si Schumpeter (1911) introduit l'entrepreneur dans l'analyse, comme un individu isolé qui puise dans un stock d'inventions préexistantes, il faut attendre Kirzner (1973) pour voir émerger une première analyse du processus de découverte d'opportunités par l'entrepreneur: l'entrepreneur est alors un individu doté d'une qualité particulière, la «vigilance» (entrepreneurial alertness). Dans cette perspective, des travaux empiriques se sont multipliés, cherchant à opérationnaliser ce cadre théorique et à repérer les caractéristiques individuelles favorisant la vigilance et l'esprit d'entreprise. Mais cette analyse des différences d'aptitudes ou de comportement des entrepreneurs par rapport aux non-entrepreneurs est apparue comme restrictive, voire comme une impasse (Gartner, 1988). Plus encore, les études ont fait ressortir que la reconnaissance d'opportunité est un processus qui repose généralement sur des échanges dans le temps entre l'entrepreneur et son entourage (Hills, 1995; Hills, Lumpkin et Singh, 1997).

L'importance attestée des relations sociales de l'entrepreneur apparaît en résonance avec des travaux menés en sociologie dans le cadre de la théorie des réseaux sociaux (TRS). Cette théorie, qui fait l'objet d'un intérêt croissant dans les sciences de gestion (Huault, 2002; Academy of Management, 2002), a également suscité nombre de travaux focalisés sur l'analyse du processus entrepreneurial, chez ses pères fondateurs (Burt, 1992, 2000b; Granovetter, 1995; Swedberg, 2000), comme chez leurs continuateurs. Hoang et Antoncic (2002) font ainsi état de plus de 70 études appliquant la TRS à l'entrepreneuriat. Nous montrerons, dans cet article, comment la TRS peut, au-delà d'une simple analyse de l'entrepreneur, permettre d'affiner l'analyse de la reconnaissance d'opportunité.

À cette fin, après avoir souligné combien les travaux historiques réalisés par l'école autrichienne (Kirzner et ses épigones) ou par les pionniers de l'étude de la reconnaissance d'opportunité (essentiellement au sein du Babson College) mènent à des impasses théoriques et/ou empiriques (section 1), nous montrerons comment la TRS permet de mieux éclairer le processus de reconnaissance d'opportunité (section 2).

\section{La reconnaissance d'opportunité: une question méconnue?}

Les travaux sur l'opportunité entrepreneuriale sont anciens, cette question ayant suscité un intérêt modéré mais constant de la part des économistes et des gestionnaires. Par-delà la diversité des appartenances disciplinaires, nous proposons de

Revue internationale P.M.E., vol. 18, nº 1, 2005

(C) 2005 - Presses de l'Université du Québec

Édifice Le Delta I, 2875, boul. Laurier, bureau 450, Sainte-Foy, Québec G1V 2M2 - Tél.: (418) 657-4399 - www.puq.ca

Tiré de: Revue internationale P.M.E., vol. 18, $\mathrm{n}^{\circ} 1$, sous la direction de Louis Raymond • PME1801N

Tous droits de reproduction, de traduction et d'adaptation réservés 
distinguer deux approches du phénomène: une approche autrichienne, dans la lignée des travaux des économistes, qui souffre d'une position «en surplomb» (Simon, 1983) et d'un manque de vérification empirique, et une approche empiriste, qui paraît biaisée par l'absence d'une réflexion fondamentale sur la nature de l'opportunité entrepreneuriale.

\subsection{L'école autrichienne: une vision soudaine et individuelle de la reconnaissance d'opportunité}

Une avancée théorique majeure sur la prise en compte de la reconnaissance d'opportunité est effectuée par l'économiste Kirzner, représentant éminent de l'école autrichienne, avec l'introduction du concept d' «alertness». Son approche, qui permet une première analyse du processus de reconnaissance d'opportunité, a suscité des travaux ayant pour objectif une opérationnalisation des concepts et ainsi un éclairage concret du phénomène.

\subsubsection{Le cadre conceptuel de Kirzner: l'entrepreneur vigilant}

Kirzner (1973) justifie l'existence des opportunités de marché par une remise en cause radicale de l'hypothèse de transparence de l'information qui est au cœur du modèle classique de la concurrence parfaite. En effet, dans la réalité, les acteurs du marché sont loin d'être omniscients, autrement dit, leur connaissance des conditions de marché n'est pas parfaite. C'est cette situation qui explique l'existence d'une multitude d'opportunités à un moment donné. Ces opportunités sont de deux types: il s'agit, d'une part, «des vendeurs qui risquent de vendre à des prix plus faibles que ce qu'ils auraient pu obtenir (notamment en raison du fait qu'ils proposent des produits moins demandés par les consommateurs, alors que des produits davantage valorisés par le marché auraient pu être obtenus avec des ressources identiques)». Il s'agit, d'autre part, «des acheteurs qui risquent d'acheter à des prix plus élevés que nécessaire (notamment parce que les produits achetés ont consommé des ressources plus coûteuses que d'autres ressources qui auraient permis d'obtenir les mêmes produits» (Kirzner, 1973, p. 41).

La reconnaissance de ces opportunités de marché constitue donc la raison d'être de celui que Kirzner appelle «l'entrepreneur pur». Pour ce faire, Kirzner décrit l'entrepreneur par une qualité psychologique particulière, à savoir l'«alertness», que l'on pourrait traduire par «vigilance». La vigilance est cette compétence développée au plus haut point chez l'entrepreneur et qui, plus que les autres, lui permet de savoir où se trouve l'information pertinente pour découvrir des opportunités de marché. «En dernière analyse, le type de connaissance nécessaire pour l'entrepreneuriat est le fait de savoir où chercher l'information plutôt que le fait de disposer d'informations substantielles sur le marché. Il semble que le mot

Revue internationale P.M.E., vol. 18, nº 1, 2005

(C) 2005 - Presses de l'Université du Québec

Édifice Le Delta I, 2875, boul. Laurier, bureau 450, Sainte-Foy, Québec G1V 2M2 • Tél.: (418) 657-4399 - www.puq.ca

Tiré de: Revue internationale P.M.E., vol. 18, $\mathrm{n}^{\circ} 1$, sous la direction de Louis Raymond $\cdot$ PME1801N

Tous droits de reproduction, de traduction et d'adaptation réservés 
vigilance est le terme qui exprime le mieux ce type de connaissance» (Kirzner, 1973, p. 68). L'entrepreneur est celui qui, par arbitrage, corrige ces écarts du marché. En fait, l'entrepreneur se définit principalement comme celui qui sait identifier les occasions de profit: «C'est l'identification réussie des finalités et des moyens, plutôt que l'utilisation efficiente des ressources, qui conduit aux bonnes décisions concernant la qualité des produits» (Kirzner, 1973, p. 139).

Le problème fondamental de la reconnaissance d'opportunité de marché est que l'on ne sait pas a priori ce que l'on recherche. Si tel n'était pas le cas, le marché aurait déjà réalisé l'arbitrage. D'où les questions fondamentales suivantes: comment peut-on être à la recherche d'un objet non clairement défini et dont l'existence même pose problème? Comment reconnaître cet objet si tant est qu'on le trouve? La procédure de recherche ne peut pas reposer sur la rationalité économique traditionnelle qui effectue un calcul coût-bénéfice en comparant les coûts liés à la recherche d'information aux gains attendus par l'obtention d'une information plus précise. La découverte d'opportunité de marché ne peut donc se faire que de manière spontanée et aléatoire; elle repose entièrement sur la capacité d'un individu, l'entrepreneur, à reconnaître dès qu'elles se présentent des occasions de profit que les autres ne voient pas.

\subsubsection{Les validations empiriques: opérationnaliser pour éclairer la reconnaissance d'opportunité}

Parmi les études empiriques qui ont utilisé les travaux de Kirzner (Long et McMullan, 1984; Hills, 1995), ce sont les travaux de Kaish et Gilad (1991) et ceux de Caglio et Taub (1992) qui vont le plus loin dans l'opérationnalisation du concept de «vigilance entrepreneuriale». Ces deux travaux cherchent à mettre en évidence une attitude spécifique des entrepreneurs, attitude caractéristique de leur «vigilance». Il s'agit, en effet, de caractériser les éléments distinctifs de la «vigilance entrepreneuriale», en comparant l'attitude de l'entrepreneur avec celle d'une population témoin de cadres d'entreprise.

Kaish et Gilad (1991) vont alors opérationnaliser la vigilance en élaborant une série d'hypothèses relatives à la façon dont pense et agit l'entrepreneur. Le degré de vigilance entrepreneuriale est alors positivement corrélé à:

- la part de temps libre consacré à la réflexion individuelle en vue d'améliorer la marche des affaires;

- la quantité et la variété des lectures solitaires, qu'elles concernent ou non les affaires;

- la propension à orienter les conversations de soirée sur des sujets ayant trait aux affaires;

Revue internationale P.M.E., vol. 18, $\mathrm{n}^{\circ} 1,2005$

(C) 2005 - Presses de l'Université du Québec

Édifice Le Delta I, 2875, boul. Laurier, bureau 450, Sainte-Foy, Québec G1V 2M2 • Tél.: (418) 657-4399 - www.puq.ca

Tiré de: Revue internationale P.M.E., vol. 18, $\mathrm{n}^{\circ} 1$, sous la direction de Louis Raymond • PME1801N

Tous droits de reproduction, de traduction et d'adaptation réservés 
- la faculté de nouer des contacts potentiellement utiles en toutes circonstances (notamment en engageant la conversation avec des étrangers rencontrés lors des déplacements professionnels, dans les trains ou encore les avions).

Il est alors possible de tester ces hypothèses à partir d'une enquête par questionnaires menée auprès de 51 créateurs d'entreprise et 36 cadres d'un grand établissement financier. Les résultats font ressortir que les entrepreneurs s'exposent à beaucoup plus d'informations que les cadres.

En outre, leur vigilance s'exerce dans des lieux plus inhabituels: à la différence du manager, l'entrepreneur sera vigilant en dehors de son temps et de son lieu de travail habituels. En fait, la vigilance s'apparente avant tout à un état d'esprit de l'entrepreneur qui l'incite finalement à ramener tout ce qu'il voit ou entend à son affaire, et cela, de manière continue sans qu'il existe de cloisonnement strict entre vie privée et vie professionnelle. L'entrepreneur semble donc en quelque sorte toujours en éveil, ce qui apparaît comme le prix à payer pour être en mesure de saisir une opportunité au moment où elle se présente.

Si Kaish et Gilad (1991) s'en tiennent à mettre en évidence une attitude spécifique des entrepreneurs dans leurs rapports à l'information, Caglio et Taub (1992) s'efforcent de relever la spécificité des processus mentaux des entrepreneurs leur permettant de convertir des informations inexploitées par d'autres en opportunité de marché. Leur étude, de type exploratoire, porte sur une population relativement limitée et composée de quatre cadres et de quatre créateurs d'entreprise. Il leur est proposé d'analyser une étude de cas suffisamment ambiguë dans une optique de découverte d'opportunité de marché. Les individus sont interrogés une vingtaine de minutes et il leur est demandé de raisonner à haute voix. Les auteurs procèdent ensuite par analyse du discours. Leur étude montre que lorsqu'on soumet aux cadres et aux créateurs d'entreprise des situations d'entreprises ambiguës, ils ne raisonnent pas de la même manière. En fait, on observe deux différences importantes:

- les deux groupes diffèrent dans leur façon de poser les problèmes. Les créateurs d'entreprise ont une approche plus personnelle des problèmes, ils s'appuient volontiers sur leur propre expérience pour organiser leur réflexion. Les cadres, quant à eux, passent davantage de temps à essayer d'utiliser différentes grilles d'analyse proches de celles que l'on pourrait trouver dans de bons manuels de gestion;

- les créateurs d'entreprise ont besoin d'une information plus contextualisée, c'est-à-dire plus précise pour mener leur raisonnement. Les cadres, quant à eux, peuvent spéculer sur des informations ayant un caractère plus général.

En définitive, à travers ces deux études empiriques, l'approche de Kirzner est centrée sur les processus cognitifs (et psychologiques) du créateur d'entreprise.

Revue internationale P.M.E., vol. 18, nº 1, 2005

(C) 2005 - Presses de l'Université du Québec

Édifice Le Delta I, 2875, boul. Laurier, bureau 450, Sainte-Foy, Québec G1V 2M2 • Tél.: (418) 657-4399 - www.puq.ca

Tiré de: Revue internationale P.M.E., vol. 18, $\mathrm{n}^{\circ} 1$, sous la direction de Louis Raymond $\cdot$ PME1801N

Tous droits de reproduction, de traduction et d'adaptation réservés 
Une telle optique suffit-elle à épuiser la question? On sent bien en effet qu'il faut également considérer ce qui se passe autour de l'entrepreneur et qui apparaît d'ailleurs dans les opérationnalisations du concept de vigilance de Kirzner. Cependant, ces aspects ne sont que cités sans faire l'objet d'une étude systématique. Il s'agit notamment de la propension à orienter les conversations de soirée sur des sujets ayant trait aux affaires et de la faculté de nouer des contacts potentiellement utiles en toutes circonstances.

Les travaux empiriques en gestion vont, du reste, insister sur ces lacunes et susciter des interrogations sur la nécessité de changer l'analyse du phénomène.

\subsection{La reconnaissance d'opportunité en gestion: des résultats empiriques appelant des questionnements nouveaux}

Les gestionnaires ont développé des travaux visant à mieux cerner le processus de reconnaissance d'opportunité, en se situant tous par rapport à l'analyse de Kirzner. Ce faisant, ils ont pu souligner le rôle central joué par les relations interpersonnelles dans la reconnaissance des opportunités de marché. Cet accent, s'il souligne la limitation de la perspective kirznérienne, ouvre la voie à l'application de nouveaux outils théoriques, lesquels permettent une meilleure prise en compte de l'inscription de l'entrepreneur dans des réseaux sociaux.

En sciences de gestion, le thème de la découverte d'opportunité de marché apparaît dès le milieu des années 1980 et, depuis lors, fait régulièrement l'objet de publications. Cependant la plupart des recherches (Long et McMullan, 1984; Koller, 1988; Teach, Schwartz et Tarpley, 1989; Hills, 1995) sont de nature descriptive et exploratoire. En effet, il s'agit souvent, à l'aide de questionnaires soumis à des chefs d'entreprise, d'essayer de mieux cerner la façon dont ces derniers perçoivent la découverte des opportunités de marché, afin de mieux comprendre cette phase préalable à la création d'entreprise. Plusieurs constats ressortent de ces études.

D'une part, l'accent est mis sur une dimension essentielle de processus de création d'entreprise, à savoir le rôle des relations interpersonnelles dans la phase de détection des opportunités de marché. En effet, pour Koller (1988), la moitié des 82 entrepreneurs interrogés déclaraient que leurs idées de création leur furent suggérées par quelqu'un d'autre. Ce résultat empirique est confirmé par Hills, Lumpkin et Singh (1997), pour qui la moitié des entrepreneurs de leur échantillon de 171 personnes peuvent être qualifiés d'entrepreneurs-réseau. Pour ces derniers, la découverte de l'opportunité de marché est à mettre au crédit de leur réseau social personnel. Les autres entrepreneurs étant qualifiés par Hills de «solo entrepreneurs». Pour eux en revanche, la découverte de l'opportunité de marché ne résulte que de leur propre action. On conçoit l'écart avec l'analyse individuelle à laquelle procédait l'école autrichienne. Ici, cette situation correspond au mieux à un cas particulier.

Revue internationale P.M.E., vol. 18, nº 1, 2005

(C) 2005 - Presses de l'Université du Québec

Édifice Le Delta I, 2875, boul. Laurier, bureau 450, Sainte-Foy, Québec G1V 2M2 - Tél.: (418) 657-4399 - www.puq.ca

Tiré de: Revue internationale P.M.E., vol. 18, $\mathrm{n}^{\circ} 1$, sous la direction de Louis Raymond • PME1801N

Tous droits de reproduction, de traduction et d'adaptation réservés 
D'autre part, Hills (1995) et Hills, Lumpkin et Singh (1997) soulignent, quant à eux, que la reconnaissance d'opportunité par l'entrepreneur ne provient pas d'une illumination soudaine. À la différence de la vision de Kirzner qui suppose l'instantanéité de la découverte, ces auteurs insistent sur la dimension processuelle de la reconnaissance d'opportunité. Pour plus de $91 \%$ des entrepreneurs, la reconnaissance d'opportunité a nécessité plusieurs étapes d'apprentissage (Hills, Lumpkin et Singh, 1997, p. 176), qu'il s'agisse d'entrepreneurs inscrits dans une démarche strictement individuelle ou bien d'entrepreneurs s'appuyant sur des réseaux de contacts pour découvrir une opportunité de marché. Dans le même ordre d'idées, l'étude longitudinale réalisée par Gasse, Diochon et Menzies (2002) auprès d'un échantillon de 112 entrepreneurs montre que « $78 \%$ des répondants ont mentionné avoir réfléchi longtemps avant de démarrer leur projet d'entreprise; en fait, plus de $67 \%$ auraient réfléchi pendant plus de 36 mois. Seulement $19 \%$ prétendent que l'idée de démarrer une entreprise est apparue soudainement comme ça» (Gasse, Diochon et Menzies, 2002, p. 6). La perception d'une opportunité par l'entrepreneur s'inscrit donc généralement au cours du temps et de discussions multiples.

Nous sommes ainsi en présence d'un faisceau convergent d'études empiriques qui mettent en évidence le rôle crucial des relations interpersonnelles dans le processus de découverte des opportunités de marché. Ces premiers résultats ne font-ils pas que corroborer le postulat théorique de Granovetter (1985) qui, avec son concept de «l'encastrement» (embeddedness), souligne le rôle déterminant des relations personnelles concrètes et des structures de ces relations dans la compréhension des comportements économiques?

Pour analyser de façon convaincante l'action humaine, il faut éviter l'atomisation qui est implicite dans les deux positions théoriques extrêmes, sous- et sursocialisées. Les acteurs ne se comportent pas et ne prennent pas leurs décisions comme des atomes indépendants de tout contexte social, pas plus qu'ils ne suivent docilement un scénario écrit pour eux et qui serait fonction de l'ensemble des catégories sociales auxquelles ils appartiennent. Au contraire, les actions qu'ils entreprennent pour atteindre un objectif sont encastrées dans des systèmes concrets, continus de relations sociales (Granovetter, 1985).

C'est précisément sur ces systèmes concrets, continus de relations sociales que nous souhaitons focaliser notre attention dans le cadre des comportements de vigilance de l'entrepreneur en quête d'opportunités de marché. Nous nous inscrivons donc pleinement dans la perspective de Aldrich et Zimmer (1986, p. 4) qui soutiennent que «l'entrepreneuriat est encastré dans un contexte social, guidé et facilité ou contraint et entravé par la position des acteurs au sein de réseaux sociaux».

Dès lors, il devient légitime de se poser la question principale de recherche suivante: quel est le rôle des réseaux sociaux dans le processus d'identification des opportunités de marché par le créateur d'entreprise? Et, dans quelle mesure la TRS est de nature à conduire vers une meilleure perception de la reconnaissance d'opportunité?

Revue internationale P.M.E., vol. 18, $n^{\circ}$ 1, 2005

(C) 2005 - Presses de l'Université du Québec

Édifice Le Delta I, 2875, boul. Laurier, bureau 450, Sainte-Foy, Québec G1V 2M2 • Tél.: (418) 657-4399 - www.puq.ca

Tiré de: Revue internationale P.M.E., vol. 18, $\mathrm{n}^{\circ} 1$, sous la direction de Louis Raymond $\cdot$ PME1801N

Tous droits de reproduction, de traduction et d'adaptation réservés 


\section{Théorie des réseaux sociaux et opportunités de marché}

Les études empiriques ont révélé à la fois la dimension collective et processuelle de la recherche d'opportunité. Il est alors nécessaire de s'interroger sur l'éclairage que permet la TRS sur cette phase. Il convient de se livrer à une analyse précise de son déroulement, en distinguant le rôle joué par les réseaux dans la détection d'opportunité et dans son évaluation, ce qui permettra de lever les apories d'une vision individualiste et statique de la recherche d'opportunité par l'entrepreneur.

À cette fin, il sera possible de nous interroger sur le rôle joué par les réseaux sociaux dans les différentes phases du projet entrepreneurial, plus particulièrement dans celle de reconnaissance d'opportunité (détection et évaluation; figure 1). Pour cela, nous nous appuierons notamment sur les travaux fondateurs de Ronald Burt et de Mark Granovetter ${ }^{2}$, afin d'étudier ces deux moments du projet de création d'entreprise.

FIGURE 1

\section{Les phases du projet entrepreneurial}

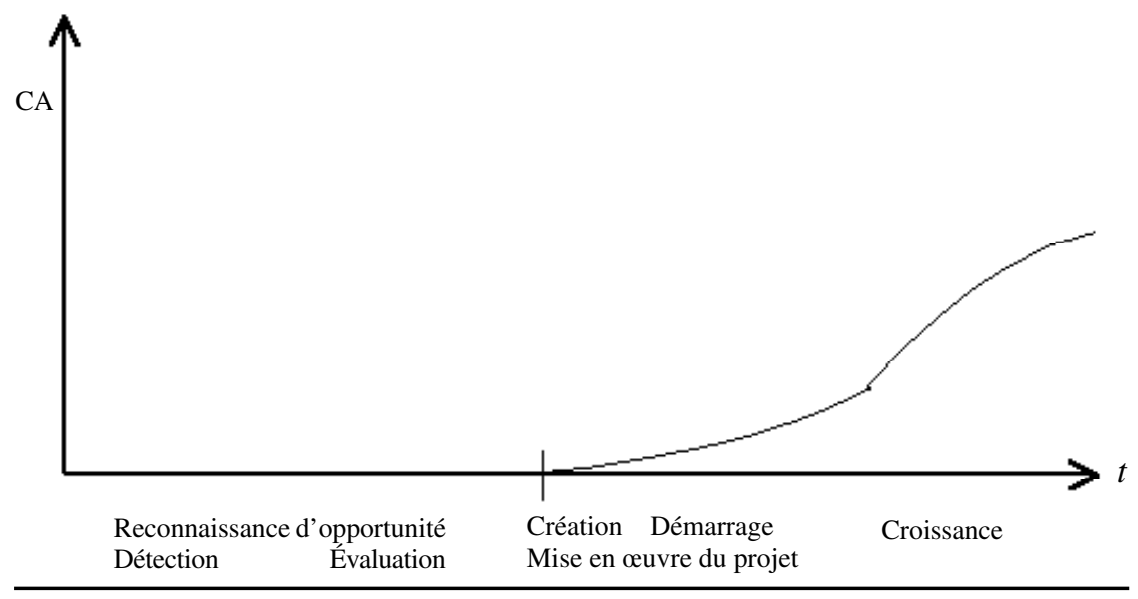

2. Burt est parfois opposé à Granovetter dans la mesure où l'entrepreneur a, chez lui, une vision instrumentale du réseau, décidant de "gérer» ses contacts en fonction de ses besoins (Granovetter, 2001, p. 4; Ferrary, 2002, p. 70). Les deux analyses demeurent, cependant, complémentaires dans l'analyse du phénomène entrepreneurial et mobilisent les mêmes outils analytiques (Lévesque, Bourque et Forgues, 2001).

Revue internationale P.M.E., vol. 18, $\mathrm{n}^{\circ}$ 1, 2005

(C) 2005 - Presses de l'Université du Québec

Édifice Le Delta I, 2875, boul. Laurier, bureau 450, Sainte-Foy, Québec G1V 2M2 • Tél.: (418) 657-4399 - www.puq.ca

Tiré de: Revue internationale P.M.E., vol. 18, $\mathrm{n}^{\circ} 1$, sous la direction de Louis Raymond PME1801N

Tous droits de reproduction, de traduction et d'adaptation réservés 


\subsection{Les réseaux sociaux, facteur de reconnaissance d'opportunité}

Dès l'origine, la TRS a été utilisée pour examiner la question de la mobilisation des réseaux pour accéder à des opportunités. Cependant, alors que les premiers travaux s'intéressaient à la reconnaissance d'opportunités d'emplois, des travaux ultérieurs comme ceux de Burt (1992 et 2000a) et Granovetter (1995) ont ouvert une fenêtre sur le rôle des réseaux dans la reconnaissance des opportunités entrepreneuriales.

Les travaux de Mark Granovetter marquent, avec ceux de White dont il a été l'élève, le début des travaux sur la TRS $^{3}$. Granovetter insistait, dès ses travaux de thèse sur le marché du travail, sur «la force des liens faibles» (1973), mettant en relief le rôle qu'entretiennent les liens faibles (comprendre des relations épisodiques) pour saisir la dynamique des actions individuelles ou l'organisation des communautés. Il montre ainsi, à travers une importante étude empirique, comment la recherche d'emploi est facilitée par le fait d'avoir des «relations», des contacts, avec des personnes qui sont dans des réseaux connexes des réseaux habituels (Granovetter, 1974). Les liens faibles permettent ainsi d'accéder à des informations (ici sur des offres d'emplois) possédées dans des réseaux connexes des réseaux habituels des individus. Au contraire, des personnes qui évoluent au sein de réseaux relationnels fractionnés, n'entretenant pas de liens faibles, mais uniquement baignées dans des liens forts, ne parviendront que difficilement à accéder à de nouveaux emplois. La diffusion de l'information mais aussi l'action collective seront ainsi influencées par la structure des réseaux, la densité des liens et le cloisonnement entre les réseaux sociaux.

On conçoit aisément la transposition réalisable lorsqu'on s'intéresse à la compréhension de l'entrepreneuriat. Il pourrait s'agir de voir dans quelle mesure les «liens faibles» contribuent à la recherche d'opportunité. Burt (1992, 2000a) permet d'aller au-delà dans la précision de l'analyse, en introduisant le concept de «trou structurel» (structural hole). L'entrepreneur est défini comme situé à l'intersection de trous structuraux, c'est-à-dire de réseaux de contacts non redondants. Dans ce cas, l'individu est «capable d'ajouter de la valeur en faisant l'intermédiaire entre les différents réseaux» (Burt, 1992, p. 34-36; 2000a, p. 11), ou «en construisant des ponts entre les trous structuraux» (Burt, 2000a, p. 29). Dès lors, deux aspects sont à souligner:

- d'une part, «les individus qui vivent à une intersection de mondes sociaux ont plus de chances d'avoir de bonnes idées» (Burt, 2002, p. 1). Dans la logique de Burt, en étant en contact avec des résaux distincts, l'individu est confronté à des façons de voir différentes, il a connaissance des manques des divers réseaux, ce qui lui permet d'être en position idéale pour innover;

3. Ce paragraphe prend particulièrement appui sur Chabaud (2002) et Steiner (2002).

Revue internationale P.M.E., vol. 18, $n^{\circ}$ 1, 2005

(C) 2005 - Presses de l'Université du Québec

Édifice Le Delta I, 2875, boul. Laurier, bureau 450, Sainte-Foy, Québec G1V 2M2 • Tél.: (418) 657-4399 - www.puq.ca

Tiré de: Revue internationale P.M.E., vol. 18, $\mathrm{n}^{\circ} 1$, sous la direction de Louis Raymond $\cdot$ PME1801N

Tous droits de reproduction, de traduction et d'adaptation réservés 
- d'autre part, cette innovation est valorisable car l'entrepreneur, par sa position, perçoit avant les autres l'intérêt d'une intermédiation ${ }^{4}$. Dans la vision de Burt, la position de «go-between» permet à l'entrepreneur de retirer de la valeur de son rôle d'intermédiaire, qu'il s'agisse de bénéfices informationnels (accès à une information non perçue par les membres des réseaux, perception de sa valeur) et des bénéfices liés à un meilleur contrôle de l'information ( $c f$. Burt, 1993).

Si l'on perçoit l'intérêt d'un tel éclairage, plusieurs questions ressortent néanmoins:

- Quelle est la fréquence du recours aux réseaux pour reconnaître l'opportunité de marché? Nous avons vu dans la section précédente qu'une partie seulement des entrepreneurs déclare s'appuyer sur des réseaux pour détecter l'opportunité de marché. Une exploration précise est donc requise.

- Une fois cette question de la fréquence réglée, la question devient celle de la nature des liens mobilisés pour détecter l'opportunité de marché. Peut-on alors transposer la proposition de Granovetter (1974) concernant le marché de l'emploi au domaine de la découverte d'opportunité de marché ? La proposition deviendrait: il existe une prédominance des liens faibles dans les communications d'informations sur les opportunités de marché, ce qui paraît cohérent avec l'analyse de Burt (1992). Ou bien, au contraire, l'entrepreneur trouve-t-il son inspiration dans des liens forts (fussent-ils non redondants)? Cette deuxième interrogation permettrait de tester tout à la fois les analyses de Granovetter et de Burt.

Si l'on a jusqu'alors insisté sur l'importance possible des réseaux sociaux dans la détection d'opportunité de marché, il convient d'observer que l'analyse a été réalisée en supposant que la phase de détection se suffit à elle-même. Or, ainsi que Hills, Lumpkin et Singh (1997) l'ont soutenu, le lancement d'un projet passe certes par la détection d'une opportunité, mais aussi par une phase d'évaluation ou de validation, pour en cerner la faisabilité et l'intérêt.

\subsection{Les réseaux sociaux, aide à l'évaluation des opportunités de marché}

La création d'entreprise est une activité risquée. Si elle repose sur la détection d'opportunité, il convient de signaler que l'entrepreneur va chercher à valider ses choix, à évaluer l'opportunité perçue avant de chercher à créer son entreprise. Plus

4. Il ne s'agit pas de réduire l'entrepreneur à un simple intermédiaire mais de montrer l'importance des trous structuraux dans l'accès à l'information et aux ressources.

Revue internationale P.M.E., vol. 18, n 1, 2005

(C) 2005 - Presses de l'Université du Québec

Édifice Le Delta I, 2875, boul. Laurier, bureau 450, Sainte-Foy, Québec G1V 2M2 - Tél.: (418) 657-4399 - www.puq.ca

Tiré de: Revue internationale P.M.E., vol. 18, $\mathrm{n}^{\circ} 1$, sous la direction de Louis Raymond • PME1801N

Tous droits de reproduction, de traduction et d'adaptation réservés 
précisément, les observations empiriques montrent que l'idée de l'entrepreneur mûrit et que l'opportunité de marché (et le projet) n'émerge généralement pas en une seule fois, mais qu'il y a au contraire des allers-retours sur l'idée et le projet, des tests et validations successifs. Ainsi, les phases de détection et de validation, loin d'être séquentielles, sont au contraire finement entremêlées. Pour faciliter l'exposé, nous supposerons cependant une démarcation entre ces deux phases qui diffèrent au moins sur le plan logique. Dès lors, l'entrepreneur potentiel va-t-il s'appuyer sur des réseaux pour effectuer l'évaluation de l'opportunité de marché? Et, dans l'affirmative, à quels réseaux va-t-il faire appel?

La découverte d'opportunité de marché par l'entrepreneur est un processus et non pas une illumination soudaine (Hills, Lumpkin et Singh, 1997). En outre, toujours d'après Hills, la majorité des entrepreneurs s'en remettent volontiers à l'intuition plutôt qu'à des études de marché pour évaluer une opportunité de marché, notamment en raison de contraintes financières certaines. Si l'intuition joue un rôle aussi important dans l'évaluation, on peut raisonnablement supposer que cette intuition de l'entrepreneur se forge également au contact de nombreux avis sollicités dans le cadre de son réseau relationnel. Il est alors intéressant de chercher à préciser l'utilité du recours aux réseaux dans cette phase de validation de l'opportunité de marché.

Or, la détection et la validation des opportunités de marché sont deux phases ayant des caractéristiques différentes. La détection requiert que l'entrepreneur puisse accéder à des informations, à des manières de penser et de voir distinctes, afin de percevoir des opportunités. Ainsi, le concept de trou structurel de Burt, mais aussi celui de liens faibles de Granovetter paraissent stimulants dans la mesure où ils introduisent explicitement l'idée d'accès à des réseaux non redondants et, donc, à des informations distinctes. La phase de validation de l'opportunité de marché est tout autre. L'entrepreneur, qui possède une idée va en effet chercher à la tester auprès de diverses personnes et instances. Cette phase d'évaluation est délicate. En effet, l'entrepreneur potentiel a besoin d'accéder à des avis informés, à des avis complémentaires du sien, tout en évitant de transmettre les informations sur son projet à des individus pouvant potentiellement entrer en compétition avec lui. La question de la confiance en l'interlocuteur joue ici un rôle crucial.

Trois recours différents sont possibles:

- d'une part, l'entrepreneur peut s'appuyer sur ses liens forts. La confiance mise dans la famille et les proches permet en effet de s'adresser à eux en limitant des problèmes d'opportunisme. On conçoit alors la facilité du dialogue entre l'entrepreneur et son interlocuteur, mais aussi les limitations (problème de la proximité cognitive au sein d'un réseau dense et problèmes de compétence des acteurs approchés);

Revue internationale P.M.E., vol. $18, \mathrm{n}^{\circ} 1,2005$

(C) 2005 - Presses de l'Université du Québec

Édifice Le Delta I, 2875, boul. Laurier, bureau 450, Sainte-Foy, Québec G1V 2M2 • Tél.: (418) 657-4399 - www.puq.ca

Tiré de: Revue internationale P.M.E., vol. 18, $\mathrm{n}^{\circ}$ 1, sous la direction de Louis Raymond • PME1801N

Tous droits de reproduction, de traduction et d'adaptation réservés 
- d'autre part, l'entrepreneur peut s'appuyer sur des liens faibles. Il peut mobiliser des relations acquises notamment dans son expérience (professionnelle ou autre) passée, afin de soumettre à la critique son projet devant des interlocuteurs qui possèdent des connaissances et des positions complémentaires aux siennes;

- enfin, l'entrepreneur peut s'adresser à des professionnels. Malgré le coût possible de ces démarches, qui est à souligner et peut en réduire l'intérêt (Hills, Lumpkin et Singh, 1997), il convient d'insister sur l'importance d'un accès à des professionnels de la création d'entreprise. Notons, sur ce point, que les pouvoirs publics ont favorisé la mise en place de réseaux d'accompagnement destinés à valider et à éclairer les choix des entrepreneurs (Chabaud, Ehlinger et Perret, 2004; CSES, 2002). Comme le relevait un directeur d'incubateur, l'entrepreneur trouve dans l'incubateur ce qu'il ne s'attendait pas à trouver. Il attend généralement des subventions et des locaux à bas prix, il trouve en fait un coaching et un regard critique sur son projet, le directeur d'incubateur estimant que son rôle est de «tester» le projet de l'entrepreneur, de l'analyser finement afin d'en évaluer la pertinence. Selon l'étude de Dussault et al. (1996) réalisée auprès de 88 entrepreneurs québécois et 69 entrepreneurs français ayant créé une entreprise dans le secteur des services depuis moins de 16 mois, il apparaitt que ces structures d'accompagnement semblent appelées à jouer un rôle croissant. En effet, c'est près de un entrepreneur sur deux qui indique avoir été soutenu par de tels réseaux d'accompagnement lors de la phase de prédémarrage de ses activités.

Ainsi, la phase d'évaluation de l'opportunité de marché paraît pouvoir, elle aussi, faire appel aux réseaux sociaux et nécessiter la réalisation d'une étude empirique précise sur ce point. Plus largement, au terme de cette analyse de la phase de reconnaissance des opportunités de marché, il semble important de noter que l'entrepreneur va déployer son projet au fil du temps et que la question de la constitution du réseau par l'entrepreneur va se poser. L'entrepreneur, confronté à un besoin d'informations et de connaissances, pourra avec le temps influencer la forme de ses réseaux sociaux, ce qui nécessite d'étudier la question des principes de leur structuration.

\subsection{La structure optimale des réseaux sociaux en matière de détection des opportunités de marché}

La question change alors de nature. Au lieu de faire du réseau social une variable indépendante qui a un impact sur la qualité du travail entrepreneurial de détection et d'évaluation des opportunités de marché, il s'agit de considérer que le réseau est lui-même constitué par l'entrepreneur afin d'appuyer son effort de création

Revue internationale P.M.E., vol. 18, $\mathrm{n}^{\circ} 1,2005$

(C) 2005 - Presses de l'Université du Québec

Édifice Le Delta I, 2875, boul. Laurier, bureau 450, Sainte-Foy, Québec G1V 2M2 - Tél.: (418) 657-4399 - www.puq.ca

Tiré de: Revue internationale P.M.E., vol. 18, n 1, sous la direction de Louis Raymond • PME1801N

Tous droits de reproduction, de traduction et d'adaptation réservés 
d'entreprise ${ }^{5}$. On peut alors se demander dans quelle mesure cet intérêt pour la démarche de constitution des réseaux par l'entrepreneur ne conduit pas à ouvrir des voies renouvelées d'opérationnalisation du concept de vigilance entrepreneuriale de Kirzner.

Ronald Burt (1992, 2000a) montre ainsi l'intérêt pour un individu, entrepreneur ou non, de porter une attention particulière à ses réseaux ( $c f$. figure 2). L'individu qui possède 15 contacts directs et indirects dans la première situation (AVANT) a ainsi une structure de réseau «peu efficace». Il n'accède en effet qu'à deux réseaux non redondants (soit un trou structurel), en investissant des ressources (temps et autres) dans des contacts directs qui ne lui apportent aucune information nouvelle. Une réorganisation de son réseau de relations (APRÈS) lui permet, en ayant toujours cinq contacts directs et les coûts afférents à l'entretien de ces contacts, d'accéder à cinq réseaux distincts. «Le temps et l'énergie épargnés par le retrait des relations avec les trois autres contacts primaires $(1,3$ et 5$)$ sont réalloués à des contacts primaires dans de nouveaux groupes (clusters). Les réseaux AVANT et APRÈS sont tous deux maintenus à un coût de cinq relations primaires, mais le réseau APRÈS est nettement plus riche en trous structuraux et ainsi en bénéfices de réseaux» (Burt, 1993, p. 295). La constitution du réseau par l'entrepreneur devient ainsi une variable d'analyse pertinente, pour comprendre l'efficacité de la création d'entreprise.

Il convient de souligner la possible contribution de cette interrogation sur la constitution des réseaux par l'entrepreneur à la thématique de Kirzner. En effet, pour ce dernier, la vigilance entrepreneuriale est présentée comme la capacité à savoir où chercher l'information pertinente, soit celle susceptible de mener à l'identification d'une opportunité de marché. Or, cette capacité inclut fondamentalement une dimension relationnelle, comme l'avaient soutenu Kaish et Gilad (1991) à la suite de Kirzner. L'analyse de Burt permet d'aller au-delà et de faire état du rôle proactif de l'entrepreneur, qui va modeler ses contacts de façon à leur intégrer des individus présents dans des réseaux non redondants, porteurs de trous structuraux ${ }^{6}$.

5. Signalons qu'un tel projet est éminemment critiqué par Granovetter qui considère qu'une telle orientation confère aux individus une rationalité instrumentale proche de celle défendue par les économistes. Cette critique nous paraît non recevable, la question étant pertinente pour le gestionnaire en quête d'une meilleure compréhension du comportement de l'entrepreneur.

6. On peut dès lors se demander si la construction d'un réseau social est un moyen de créer une opportunité qui n'apparaît alors plus comme une simple donnée qu'il suffit de reconnaître. Cette question requiert une réflexion plus approfondie sur l'ontologie de l'opportunité, réflexion que nous avons engagée dans Ngijol et Chabaud (2004).

Revue internationale P.M.E., vol. 18, nº 1, 2005

(C) 2005 - Presses de l'Université du Québec

Édifice Le Delta I, 2875, boul. Laurier, bureau 450, Sainte-Foy, Québec G1V 2M2 • Tél.: (418) 657-4399 - www.puq.ca

Tiré de: Revue internationale P.M.E., vol. 18, $\mathrm{n}^{\circ} 1$, sous la direction de Louis Raymond $\cdot$ PME1801N

Tous droits de reproduction, de traduction et d'adaptation réservés 
Figure 2

\section{Une analyse comparative des réseaux}

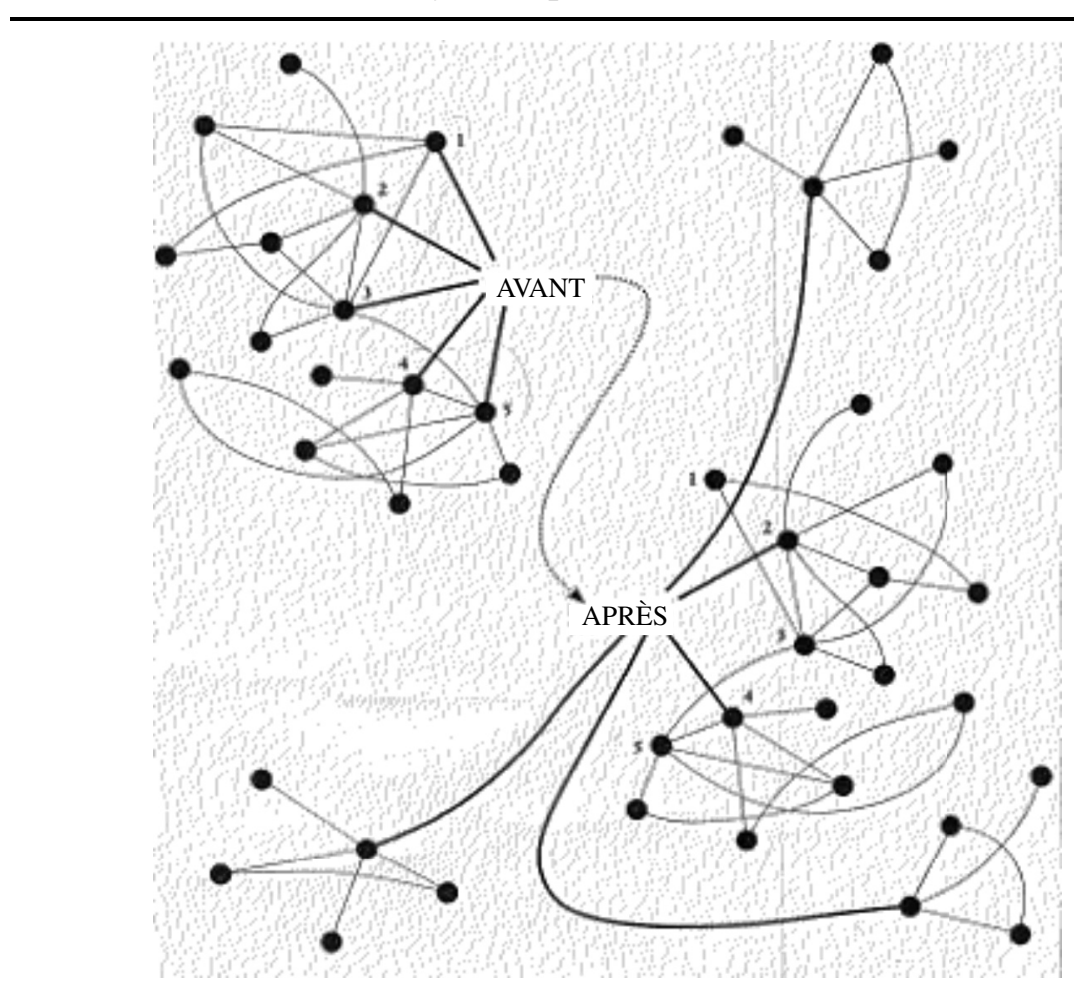

Source: Burt, 1993, p. 296.

Une telle démarche, qui a fait l'objet de peu de travaux jusqu'ici, paraît intéressante dans le cadre d'une analyse de l'entrepreneuriat ( $c f$. Hoang et Antoncic, 2002), mais elle nécessite une démarche diachronique de suivi d'une population d'individus avant même qu'ils ne s'engagent dans un rôle d'entrepreneur. On conçoit la difficulté d'un tel projet, qui exige notamment de suivre des individus sans savoir s'ils s'engageront dans une démarche entrepreneuriale et de contraster ensuite les observations effectuées sur les entrepreneurs et sur les «non-entrepreneurs». Burt a pu néanmoins fournir des éléments dans le cadre d'une étude sur une population d'individus, lui permettant notamment de contraster le rôle des réseaux chez les femmes entrepreneurs et les femmes managers, à partir d'une analyse détaillée portant sur une population de 814 étudiants diplômés d'un cursus de gestion sur une période de 60 ans (Burt, 1998, 2000b).

Revue internationale P.M.E., vol. 18, n 1, 2005

(C) 2005 - Presses de l'Université du Québec

Édifice Le Delta I, 2875, boul. Laurier, bureau 450, Sainte-Foy, Québec G1V 2M2 • Tél.: (418) 657-4399 - www.puq.ca

Tiré de: Revue internationale P.M.E., vol. 18, $\mathrm{n}^{\circ} 1$, sous la direction de Louis Raymond • PME1801N

Tous droits de reproduction, de traduction et d'adaptation réservés 
Ainsi, les réseaux sociaux jouent-ils un rôle certain dans la démarche de l'entrepreneur. Celui-ci, engagé dans un projet de création d'entreprise, va chercher à mobiliser ses réseaux pour détecter et évaluer les opportunités de marché, mais il va aussi chercher à adapter ses réseaux à ses besoins. Cependant, une étude empirique précise sera requise, afin de dépasser le stade du programme de recherche et de conforter ou discuter les quelques études empiriques existantes ( $c f$. section 1).

\section{Conclusion et voies de recherche}

Pour l'école autrichienne, l'entrepreneur est un individu vigilant qui puise dans un stock d'inventions. Or, les observations empiriques contredisent cette vision d'un entrepreneur isolé qui aurait une illumination. Sur ce point, le recours à la TRS a permis simultanément de rendre compte des critiques empiriques et d'ouvrir la voie à une analyse refondée de la reconnaissance d'opportunité par l'entrepreneur.

L'entrepreneur bénéficie de son insertion dans des réseaux relationnels et l'élaboration de son projet s'appuie sur ces réseaux pour rechercher et évaluer les opportunités de marché. Plus encore, cette démarche collective s'inscrit dans le temps et appelle des approfondissements sur la nature et les modes de mobilisation des réseaux. L'entrepreneur peut, en effet, recourir différemment aux réseaux sociaux (liens forts et/ou liens faibles) selon qu'il se préoccupe de découvrir ou d'évaluer des opportunités de marché.

Si des études empiriques récentes permettent de connecter nature des liens et degré d'innovation (Julien, Andriambeloson et Ramangalahy, 2002), voire de cerner la dynamique relationnelle de l'entrepreneur au début de son projet (Hite et Hesterly, 2001), des études complémentaires sont requises pour affiner la compréhension de la phase amont que constitue la recherche d'opportunité. Sur ce point, plusieurs axes de recherche émergent pour cerner le rôle des réseaux sociaux dans le processus de reconnaissance des opportunités de marché par le créateur d'entreprise. Si une démarche générale de cartographie des réseaux utilisés par l'entrepreneur est porteuse d'enseignements, l'interrogation sur un recours différencié aux réseaux - selon que l'entrepreneur vise à découvrir une opportunité ou à en évaluer le potentiel - permettra d'améliorer notre compréhension du comportement entrepreneurial et, plus largement, de cerner le potentiel explicatif de la TRS.

\section{Bibliographie}

Academy of Management (2002), Building Effective Networks, Colloque de Denver, Col., 9-14 août.

Aldrich, H. et C. Zimmer (1986), «Entrepreneurship through social networks», dans D.L. Sexton et R.W. Smilor (dir.), The Art and Science of Entrepreneurship, Cambridge, Mass., Ballinger.

Revue internationale P.M.E., vol. 18, $\mathrm{n}^{\circ} 1,2005$

(C) 2005 - Presses de l'Université du Québec

Édifice Le Delta I, 2875, boul. Laurier, bureau 450, Sainte-Foy, Québec G1V 2M2 • Tél.: (418) 657-4399 - www.puq.ca

Tiré de: Revue internationale P.M.E., vol. 18, $\mathrm{n}^{\circ} 1$, sous la direction de Louis Raymond $\cdot$ PME1801N

Tous droits de reproduction, de traduction et d'adaptation réservés 
Burt, R.S. (1992), Structural Holes: The Social Structure of Competition, Cambridge, Mass., Harvard Business Press.

BURT, R.S. (1993), «The network entrepreneur», dans R. Swedberg (dir.) (2000), Explorations in Economic Sociology, New York, Russel Sage Foundation.

BURT, R.S. (1998), «The gender of social capital, rationality and society», téléchargeable sur le site Web: <http://gsbwww.uchicago.edu/fac/ronald.burt/research>.

BURT, R.S. (2000a), «The network structure of social capital», dans R.I. Sutton et B. Staw (dir.), Research in Organizational Behavior, 22, Greenwich, Conn., JAI Press.

BURT, R.S. (2000b), «Creating careers: women's path through entrepreneurship», Mimeo, téléchargeable sur le site Web: <http://gsbwww.uchicago.edu/fac/ronald.burt/research>.

Burt, R.S. (2002), Social Origins of Good Ideas, Mimeo, University of Chicago.

Caglio, C.M. et R.P. TAub (1992), «Entrepreneurs and opportunity recognition», Frontiers of Entrepreneurship Research, Wellesley, Mass., Babson College.

Chabaud, D. (2002), «Granovetter et la théorie des coûts de transaction: de la controverse à la fertilisation croisée?», dans I. Huault (dir.), La construction sociale de l'entreprise, Colombelles, Éditions EMS.

Chabaud, D., S. Ehlinger et V. Perret (2004), «Les pépinières et incubateurs dans l'accompagnement de la création d'entreprises», dans C. Voisin, S. Ben Mahmoud-Jouini et S. Edouard (dir.), Les réseaux: dimensions stratégiques et organisationnelles, Paris, Economica, p. 215-227.

CSES - CENTRE FOR STRATEGY \& EVALUATION SERVICES (2002), Benchmarking of Business Incubators, rapport final, Commission européenne, Mimeo.

Dussault, L., J. Lorrain, A. Belley, B. Saporta et M. Rispal (1996), «Les activités de l'entrepreneur reliées à l'analyse et aux choix stratégiques en phase de démarrage: une étude comparative France-Québec d'entreprises de services», III Congrès international francophone sur la PME, octobre, Trois-Rivières (Québec).

Ferrary, M. (2002), «Pour une théorie de l'échange dans les réseaux sociaux», dans I. Huault (dir.), La construction sociale de l'entreprise, Colombelles, Éditions EMS.

GARTNER, W.B. (1988), «Who is an entrepreneur? Is the wrong question», American Journal of Small Business, vol. 12, $\mathrm{n}^{\circ}$ 4, p. 11-32.

Gasse, Y., M. Diochon et T.V. Menzies (2002), «Les entrepreneurs naissants et la poursuite de leur projet d'entreprise: une étude longitudinale», VI Congrès international francophone sur la PME, octobre, HEC Montréal.

Granovetter, M. (1973), «The strength of weak ties», American Journal of Sociology, vol. $78, \mathrm{n}^{\circ}$ 6, p. 1360-1380.

Granovetter, M. (1974), Getting a Job: A Study of Contacts and Careers, $2^{\mathrm{e}}$ éd. en 1995 , Chicago, University of Chicago Press.

Granovetter, M. (1985), «Economic action and social structure: the problem of embeddedness », American Journal of Sociology, vol. 91, n 3, p. 481-510.

Granovetter, M. (1995), «The economic sociology of firms and entrepreneurs», dans A. Portes (dir.), The Economic Sociology of Immigration. Essays on Networks, Ethnicity, and Entrepreneurship, New York, Russel Sage Foundation.

Revue internationale P.M.E., vol. 18, $\mathrm{n}^{\circ} 1,2005$

(C) 2005 - Presses de l'Université du Québec

Édifice Le Delta I, 2875, boul. Laurier, bureau 450, Sainte-Foy, Québec G1V 2M2 • Tél.: (418) 657-4399 - www.puq.ca

Tiré de: Revue internationale P.M.E., vol. 18, $\mathrm{n}^{\circ} 1$, sous la direction de Louis Raymond • PME1801N

Tous droits de reproduction, de traduction et d'adaptation réservés 
Granovetter, M. (2000), Le marché autrement. Les réseaux dans l'économie, Paris, Desclée de Brouwer.

GrANOvetTER, M. (2001), «A theoretical agenda for economic sociology», dans M.F. Guillen, R. Collins et P. England, Economic Sociology at the Millenium, New York, Sage.

HiLls, G.E. (1995), «Opportunity recognition by successful entrepreneurs: a pilot study», Frontiers of Entrepreneurship Research, Wellesley, Mass., Babson College.

HILLs, G.E., T.G. LuMPKIN et R.P. SINGH (1997), «Opportunity recognition: perceptions and behaviors of entrepreneurs», Frontiers of Entrepreneurship Research, Wellesley, Mass., Babson College.

Hite, J. et Y.W. Hesterly (2001), «The evolution of firm networks: from emergence to early growth of the firm», Strategic Management Journal, vol. 22, p. 275-286.

HoAng, H. et B. Antoncic (2002), «Network-based research in entrepreneurship. A critical review», Journal of Business Venturing, vol. 18, n² 2, p. 165-187.

Huault, I. (dir.) (2002), La construction sociale de l'entreprise, Colombelles, Éditions EMS.

Julien, P.-A., É. ANdriambeloson et C. Ramangalahy (2002), «Réseaux, signaux faibles et innovation technologique dans les PME du secteur des équipements de transport terrestre», $\mathrm{VI}^{\mathrm{e}}$ Congrès international francophone sur la PME, octobre, HEC Montréal.

KAISH, S. et B. GILAD (1991), «Characteristics of opportunities search of entrepreneurs versus executives: sources, interests, general alertness», Journal of Business Venturing, vol. $6, \mathrm{n}^{\circ} 1$, p. $45-61$.

KIRZNER, I.M. (1973), Competition and Entrepreneurship, Chicago, University of Chicago Press.

KoLler, R.H. (1988), «On the source of entrepreneurial ideas», Frontiers of Entrepreneurship Research, Wellesley, Mass., Babson College.

Lévesque, B., G. Bourque et E. Forgues (2001), La nouvelle sociologie économique, Paris, Desclée de Brouwer.

LONG, W. et W.E. MCMullan (1984), «Mapping the new venture opportunity identification process», Frontiers of Entrepreneurship Research, Wellesley, Mass., Babson College.

NgiJol, J. et D. Chabaud (2004), «La reconnaissance des opportunités de marché par l'entrepreneur: vers une perspective constructiviste», Cahier de recherche de DMSP, $\mathrm{n}^{\circ}$ 326, mars, Université de Paris-Dauphine.

SCHUMPETER, J.A. (1911), Der Theorie wirtschaftlichen Entwicklung, Leipzig, Dunker und Humblot, traduit en français: Théorie de l'évolution économique, Paris, Dalloz, 1935.

Simon, H.A. (1983), Reason in Human Affairs, Stanford, Cal., Stanford University Press.

STEINer, P. (2002), «Encastrement et sociologie économique», dans I. Huault (dir.), La construction sociale de l'entreprise, Colombelles, Éditions EMS.

SwEDBERG, R. (dir.) (2000), Entrepreneurship. The Social Science View, New York, Oxford University Press.

Teach, R.D., R.G. SchWARtZ et F.A. Tarpley (1989), «Recognition and exploitation of opportunity in the software industry: a study of surviving firms », Frontiers of Entrepreneurship Research, Wellesley, Mass., Babson College.

Revue internationale P.M.E., vol. 18, $\mathrm{n}^{\circ} 1,2005$

(C) 2005 - Presses de l'Université du Québec

Édifice Le Delta I, 2875, boul. Laurier, bureau 450, Sainte-Foy, Québec G1V 2M2 - Tél.: (418) 657-4399 - www.puq.ca

Tiré de: Revue internationale P.M.E., vol. 18, $\mathrm{n}^{\circ} 1$, sous la direction de Louis Raymond P PME1801N

Tous droits de reproduction, de traduction et d'adaptation réservés 